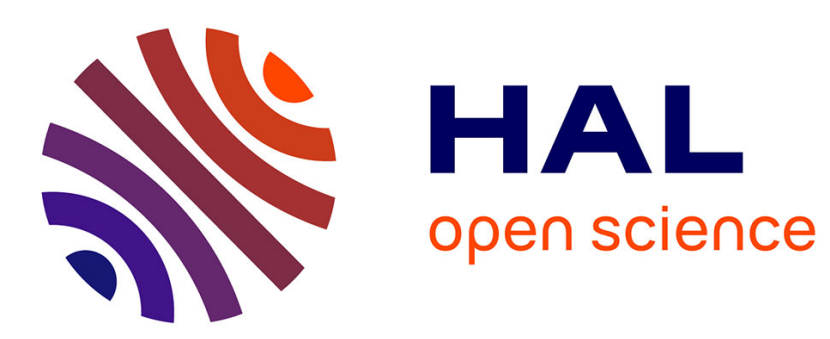

\title{
Eleusine et maïs, cent ans de cuisine des céréales chez les Rwa (Tanzanie du Nord)
}

Catherine Baroin

\section{To cite this version:}

Catherine Baroin. Eleusine et maïs, cent ans de cuisine des céréales chez les Rwa (Tanzanie du Nord). M. CHASTANET, F.-X. FAUVELLE-AYMAR et D. JUHE-BEAULATON. Cuisine et société en Afrique: histoire, saveurs, savoir-faire, Paris: Karthala, pp.265-275, 2002, Hommes et sociétés. hal-00749879

\section{HAL Id: hal-00749879 \\ https://hal.science/hal-00749879}

Submitted on 8 Nov 2012

HAL is a multi-disciplinary open access archive for the deposit and dissemination of scientific research documents, whether they are published or not. The documents may come from teaching and research institutions in France or abroad, or from public or private research centers.
L'archive ouverte pluridisciplinaire HAL, est destinée au dépôt et à la diffusion de documents scientifiques de niveau recherche, publiés ou non, émanant des établissements d'enseignement et de recherche français ou étrangers, des laboratoires publics ou privés. 


\section{Éleusine et maïs, cent ans de cuisine des céréales chez les Rwa (Tanzanie du nord)}

Catherine BAROIN

Les Rwa, agriculteurs bantous établis sur les flancs sud-est du Mont Méru, en Tanzanie du Nord, ont une alimentation assez diversifiée qui a connu une évolution considérable au fil du siècle écoulé. Celle-ci, comme celle de leurs voisins et parents les Chaga du Kilimandjaro, est liée à leur insertion progressive dans un univers économique plus large, et aux énormes changements survenus dans la société locale. Ce vaste sujet ne pouvant être abordé ici dans sa totalité, je limiterai mon propos à deux céréales, l'éleusine et le maïs, qui forment avec la banane et le haricot, depuis plus de cent ans ${ }^{1}$, la base de l'alimentation des Rwa. Tandis que le maïs, le plus souvent associé au haricot, constitue la base de la nourriture, maiis, le plus souvent associé au haricot, constitue la base de la nourriture, l'eleusine peut être consommée sous forme de porridge mais, surtout, elle
entre dans la fabrication de la bière de banane dont les Rwa absorbent entre dans la fabrica

Les Rwa, sur le Mont Méru², pratiquent une agriculture riche et très intensive où dominent le café, culture de rente, et la banane, culture vivrière. Dans un paysage très vert, à l'ombre de quelques grands arbres, se déploient les larges feuilles de la bananeraie, abritant les caféiers, sous lesquels poussent maïs et haricots. Ces deux dernières plantes, volontiers associées, occupent aussi les grands champs de la plaine plus sèche en contrebas de la montagne. Les Rwa cultivent aussi l'éleusine et bien d'autres plantes vivrières qui croissent à merveille dans cet environnement agricole très privilégié. Ils complètent leurs ressources par l'élevage

1. L. VON HÖHNEL (1968 : 152-153).

2. Ils sont connus en Tanzanie sous le nom de Meru, mais n'ont aucun rapport avec les Meru du Kenya. 
de quelques vaches laitières et de petit bétail en stabulation, auxquels sont destinés quelques champs de fourrage.

Parmi les quatre plantes alimentaires les plus importantes, seule l'éleusine est d'origine africaine. Le maïs (une variété à grains durs) est d'origine américaine de même que le haricot rouge avec lequel il est complanté ${ }^{3}$. Auparavant, les Rwa cultivaient une autre variété de haricot, au grain noir avec coin blanc, qui n'a pas été identifié. Quant à la banane, c'est une plante provenant d'Asie du Sud-Est ${ }^{4}$. Mais la culture de ces quatre plantes est depuis longtemps intégrée dans le paysage et la société des Rwa. Elles formaient déjà, il y a cent ans, la base de leur alimentation ${ }^{5}$. D'autres denrées s'y ajoutent aujourd'hui, sans doute d'introduction plus récente, telles que le riz, la pomme de terre, le thé bu avec du lait, sans compter la viande consommée en bien moindre quantité qu'autrefois, et une multitude de légumes et condiments divers.

\section{L’éleusine}

L'éleusine (umbi dans la langue des Rwa, le $k i-r w a^{6}$ ) additionnée d'eau constituait dans le passé une boisson réservée aux femmes. Cet usage a disparu, et l'éleusine est cultivée en premier lieu pour faire de la bière de banane (waRi wa umbi), en second lieu pour préparer une bouillie $(u R u)$ dont se nourrissent les nouvelles épouses, ou les mères qui viennent d'accoucher, bouillie que partagent leurs nombreuses visiteuses.

\section{La bière de banane et d'éleusine}

Les Rwa cultivent au moins une dizaine de variétés de bananes différentes, parmi lesquelles deux surtout sont utilisées pour la bière. L'une, iruru, se dénomme ndizi ya ng'ombe en swahili, c'est-à-dire «banane à vaches", et l'autre est la "banane douce à cochons", kisukari kya bouillir de longues heures dans de l'eau, jusqu'à ce que la couleur

3. Voir S. BAhuChEt et G. PhiLIPPSON (1998 : 90-91) ; J.-P. ChrétIEN (1998 : 222). 4. G. PHILIPPSON (1984: 137)

5. Comme en atteste en 1902 le journal de la Mission évangélique luthérienne locale Voir T. SPEAR (1995: 18).

6. Le ki-rwa est une langue à tons, mais la transcription adoptée ici est simplifiée. Les tons ne sont pas transcrits. La majuscule $R$ correspond au $r$ labialisé, tandis que le groupe $s h$ se prononce comme en anglais. devienne rouge. Le mélange est alors versé dans de vastes récipients de bois (mundi, pl. mindi) creusés dans de larges troncs, et laissé trois jours à découvert ${ }^{\top}$. Puis on ajoute de l'eau pour un jour de plus. Ensuite cette bouillie est filtrée à l'aide d'un sac de jute.

Parallèlement, on procède à la préparation de l'éleusine. Les grains sont mouillés, placés sur des feuilles de bananiers et recouverts d'autres feuilles de bananiers. Ainsi à l'abri, ils germent au bout de quelques jours, après quoi on les découvre et les laisse sécher à l'air libre, ce qui prend quelques jours de plus. Puis on les broie à la machine. Le produit obtenu est versé dans une grande marmite où il est cuit à l'eau comme du porridge.

Ensuite, le jus extrait de la bouillie de banane est ajouté au porridge d'éleusine, avec de l'eau. Ce mélange préparé le soir est laissé à reposer pendant la nuit, et la bière est prête le lendemain. Selon Moore ${ }^{8}$, les Chaga du Kilimandjaro qui sont, comme les Rwa, de grands consommateurs de bière de banane, ajoutent à ce mélange de la poudre d'écorce d'un arbre, le msesewe, après l'avoir mise à bouillir dans de l'eau, pour faire fermenter le liquide.

Cette boisson, considérée comme saine et nourrissante, est fabriquée en grande quantité, surtout par les hommes, sur le lieu même de consommation, c'est-à-dire le débit de boisson où elle est vendue le lendemain. Si la fabrication et la vente de la bière sont une activité plutôt masculine, en amont ce sont surtout les femmes qui accaparent le commerce des bananes nécessaires : elles les achètent dans les fermes, les épluchent et vendent leurs seaux de bananes épluchées aux fabricants de bière. Le prix obtenu pour un tel seau varie fortement selon la saison: modeste à la saison des pluies, il fait plus que tripler en saison sèche car, avec la chaleur, la consommation de bière est encore plus forte.

La bière de banane a une importance sociale considérable chez les Rwa. Les hommes surtout la consomment en grande quantité, chaque jour, dans les débits de boisson où ils aiment se retrouver en fin de ournée. L'enivrement masculin est une pratique ancienne dans la société rwa, que l'église luthérienne combat depuis un siècle avec un succès trè relatif.

En dehors des «bistrots » où elle est vendue et consommée, la bière de banane fait aussi l'objet de dons rituels et se partage dans les réunions masculines qui marquent la vie familiale et publique. Pour une demande en mariage, après que les premiers contacts ont été établis par la mère du jeune homme, son père rend visite au père de la jeune fille en lui apporjeune homme, son père rend visite au père de la jeune fille en lui apportant le cadeau rituel d'un demi-seau de bière de banane. Ensuite, lorsque
les fiançailles seront annoncées aux membres du clan patrilinéaire de $350)$. 8. S.F. MOORE $(1986: 350)$

9. Le seau utilisé, appelé debe, contient 4 gallons, soit 18,16 litres. 
fiancée, ce sont dix à vingt seaux de bière de banane que le jeune homme et sa famille devront leur offrir. Il y a vingt-cinq ans, la compensation ons d'animaux à certain mariage, de grandes quantités de bière de banane sont à nouveau offertes par le père du marié à ses hôtes, à moins que ses convictions religieuses ne le poussent à remplacer la bière par des sodas.

Un mois après le décès d'un père de famille, les hommes de son patrilignage se réunissent pour régler le partage de ses biens, et de la bière de banane est préparée en abondance pour la circonstance. Toutes les réunions de clan ou de lignage en général, où les hommes débatce divers problèmes et les mésententes familiales, les conflits de voisinage ou de limites de champs, sont autant d'occasions nouvelles de consommer ensemble de la bière de banane. Elle peut être offerte par le plaignant qui vient soumettre sa cause à l'assemblée, ou par tel coupable avéré d'un délit. C'est en effet ce que prévoit le droit coutumier des Rwa, consigné dans un long texte officiel rédigé en swahili ${ }^{11}$. Cette "Constitution » précise le montant des amendes à verser, sous forme d'animaux et de seaux de bière de banane, dans divers cas d'infraction, coups et blessures notamment.

Les cérémonies générationnelles, bien moins fréquentes, sont d'autres occasions de beuveries, comme je l'ai constaté en 1992. La société rwa est en effet organisée en classes générationnelles, chacune rassemblant des hommes sensiblement de même âge qui ont été circoncis au cours de la même période. La génération la plus jeune occupe la fonction de «guerrier», sur un modèle proche de celui des Maasaï, puis laisse la place au bout de quelques années à la génération nouvelle et rejoint, avec les plus anciennes, le groupe des hommes mûrs qui monopolisent fonctions de decision en grande cérémonie où, après de longs discours, divers cadeaux et insignes de pouvoir sont remis aux chefs des générations successives. Puis commencent les réjouissances : repas, boisson et danses, le tout aux frais de la génération qui se retire. Les quantités de bière de banane consommées en cette circonstance sont considérables, et l'ivresse quasi générale.

La bière de banane et d'éleusine constitue donc un élément capital de l'alimentation et de la vie sociale des Rwa. Il en est de même pour leurs voisins les Chaga, pour lesquels nombre de paiements et d'offrandes ritulles sont faits sous forme de bieret2 rituelles sont faits sous forme de bière ${ }^{12}$ indépendamment des énormes quantités consommées journellement dans les nombreux débits de boisson ${ }^{13}$.

0. B. KAAYA (1978: 11)

11. Mila na jadi za Wameru - Tanzania, voir C. BAROIN (à parâtre).

2. O.F.RAUM (1940).

13. S. F. MOORE $(1986: 351)$.

\section{La bouillie $(u R u)$}

La farine d'éleusine (non germée cette fois), cuite avec de l'eau et du lait, donne une bouillie $(u R u)$ qui est consommée en grandes quantités par les femmes dans deux circonstances particulières : après la cérémonie du mariage et après un accouchement. Cette bouillie est en effet une nourriture qui favorise la remise en forme de l'intéressée, et que ses visiteuses viennent partager rituellement avec elle.

Lors d'un premier mariage, le lendemain de la défloration, tandis que le drap maculé de sang a été lavé et mis à sécher au vu de tous, les parentes de la femme apportent un seau de farine d'éleusine pour préparer la bouillie $(u R u)$. La jeune épouse pour sa part doit rester oisive et garde a chambre pendant quatre jours. Les parentes du mari apportent, elles aussi, un seau de farine d'éleusine. Dès le matin, cette bouillie es consommée par les jeunes mariés et leurs nombreuses visiteuses, les parentes de l'épouse en offrant aux parentes du mari et vice-versa.

Plus que la jeune épouse, l'accouchée est aux yeux des Rwa un être affaibli et vulnérable. Elle garde la chambre pendant un à deux mois après la naissance et on lui réserve une alimentation particulière, c'est-àdire des nourritures «tendres», à la fois nourrissantes et digestes. Pendant les premiers jours, elle consomme de la soupe (mtori) à base de viande, de graisse et de banane. Puis, au cours des mois qui suivent, c'est la bouillie à base de farine d'éleusine, d'eau et de lait qui constitue sa nourriture attitrée. Cette bouillie est offerte en quantité généreuse à tout visiteur ou visiteuse. Les femmes, bien sûr, sont les plus nombreuses : ce sont les voisines, les amies, les parentes paternelles et maternelles du bébé. Chaque visiteur ou visiteuse apporte un cadeau et se voit servir en échange, outre l'incontournable bouillie d'éleusine, un plat à base de maïs (ndolo) ou bien du riz pilaf, ainsi que du thé au lait. Les deux grands-mères du nouveau-né, qui ont reçu leur part du mouton à queue grasse offert par le père de l'enfant, apportent l'une et l'autre un seau entier de farine d'éleusine, ainsi que du lait et de la graisse. Car la graisse, en particulier celle de la queue des moutons, est une nourriture « tendre comme la bouillie d'éleusine $u R u$, et elle est donc elle aussi très valorisé par les Rwa.

\section{Le maïs}

Le maïs (meemba), contrairement à l'éleusine et peut-être parce qu'il est d'introduction relativement récente, n'est jamais une nourriture rituelle. C'est une céréale strictement utilitaire qui entre dans de nombreuses préparations culinaires: nswa, ndolo, ngaRaRimu, ugali, kitalolo. $\mathrm{Ce}$ 
maïs à grains durs est le plus souvent décortiqué avant cuisson. À cette fin, il est mis à tremper au préalable dans de l'eau, puis, à l'aide d'un pilon et d'un mortier, la peau jaune est séparée du grain, presque blanc à l'intérieur. Toutes les recettes, sauf la pâte de maïs (ugali) préparée à partir de farine, utilisent du maïs en grains et nécessitent donc un long temps de cuisson pour l'attendrir. Les principales préparations culinaires à base de maïs sont détaillées ci-dessous.

\section{Le nswa}

Les Rwa considèrent ce plat de base, autrefois quotidien, comme celui uquel s'attache le plus intimement leur identité, même s'ils ne sont pas les seuls à le consommer. D'après eux, leurs voisins les Chaga le fon aussi, sous le nom de loshoro ${ }^{14}$. Les grains de maïs préalablement décortiqués sont mis à bouillir longuement dans de l'eau, puis on ajoute de la banane en morceaux, puis du lait à la fin de la cuisson. On peut aussi inclure des haricots, auquel cas ils sont incorporés avant la banane car il leur faut plus de temps pour cuire.

Autrefois, le nswa était conservé dans un récipient particulier, $i i R i(\mathrm{pl}$ meeRi), calebasse au long col muni d'un bouchon, que le mari pouvai garder dans sa maison personnelle, distincte de celle de sa femme. Mais ces habitations séparées, comme l'usage de ce récipient, tendent à disparaître aujourd'hui. Le nswa pouvait ainsi être consommé froid à tout moment de la journée, et cette disponibilité autorisait un visiteur rwa de passage à en réclamer à son hôte, ce qu'il n'aurait pas fait s'agissant d'un autre plat. Ce statut spécifique du nswa découle de ce qu'il est, aux yeux des Rwa, leur plat le plus caractéristique. C'est aussi parce qu'ils le jugent trop typique de leur propre culture qu'ils n'osent l'offrir à des étrangers. Il n'est jamais servi dans les repas collectifs auxquels sont susceptibles de prendre part des visiteurs extérieurs, c'est-à-dire aujourd'hui la plupart des repas collectifs, préparés lors des mariages, décès, naissances et autres manifestations sociales ou religieuses. Le plat le plus fréquent sera alors le ndolo, le riz pilaf (pilau), ou du ragoût de banane, qui sont moins marqués «ethniquement ».

14. Ce terme n'est pas noté par G.PHILIPPson (1984), qui consacre quatre pages à l'alimentation des Chaga. Selon cet auteur (1984. 171) nswa est le mot pour «farine» en $k i$-chaga, et dessigne dans le dialecte de Machamé, le plus proche du ki-rwa, la bouillie de farine d'éleusine cuite avec du lait, que les Rwa appellent $u R u$.
Le ndolo

Les grains de maïs décortiqués sont cuits longuement à l'eau. Ensuite on ajoute, éventuellement, des haricots, de la viande, puis de la tomate et de l'oignon. Ce plat est très commun. Il est consommé en famille, mai c'est aussi celui que l'on sert dans la plupart des réunions publiques mariages, funérailles, fêtes religieuses, etc. Moins cher et moins prestigieux que le riz pilaf, le ndolo sera servi aux visiteuses et le riz pilaf aux visiteurs si l'hôte n'a pas les moyens d'offrir le pilaf à tout le monde.

\section{Le ngaRaRimu}

Cette recette se prépare avec des grains de maïs non décortiqués et des haricots rouges non pilés. C'est un plat sans sauce, dur à mâcher et donc interdit aux circoncis, car ce ne serait pas bon pour leur convalescence.

\section{Le ngaRaRimu ta mangolo}

À la recette précédente, on ajoute des bananes séchées coupées en

\section{Le ngaRaRimu ta nyay}

Au ngaRaRimu sont ajoutés des «brèdes », c'est-à-dire des plantes à feuilles vertes (mchicha en swahili) coupées en morceaux qui cuisent avec le reste. Ce plat est réservé aux femmes car les hommes, en principe, ne mangent pas de légumes verts après leur circoncision.

\section{L'ugali}

Equivalent de ce que l'on nomme «la boule » ou «la pâte » en Afrique francophone, l'ugali est une pâte compacte de farine de maïs cuite à 
l'eau. C'est un plat si uniformément répandu en Tanzanie que l'on peut le considérer comme le plat «national» du pays. Chez les Rwa, l'ugali est avec de la farine de mais, désigner ailleurs une pâte de même consistance à base de farine de mil ou de manioc. Chaque ménagère porte son propre maïs à moudre, pour une somme modique, au moulin local, à moins que la farine ne soit achetée au détail ou par sac, ce qui est plus rare.

La farine de maïs est versée peu à peu dans l'eau bouillante et remuée énergiquement jusqu'à ce qu'elle forme une pâte. Cette dernière es servie accompagnée d'une sauce, d'une soupe, de viande ou de lai bouilli. Si la sauce est préparée avec des «brèdes» (nyayi), sa consommation est soumise à des restrictions, moins fortes cependant que pour le ngaRaRimu ta nyayi: les hommes, après leur circoncision, peuvent consommer ce plat dans leur foyer, mais jamais en présence d'une femme qui n'est pas leur parente. De nombreux hommes aujourd'hui ne respectent plus cet interdit.

\section{Le kitalolo}

Il s'agit d'un plat plus occasionnel, d'une nourriture «tendre» réservée aux femmes et aux jeunes garçons, avant leur circoncision. Au maïs cuit à l'eau sont ajoutés de la banane et des légumes verts (nyayi), voire des haricots ou du lait. Ce plat est préparé surtout au moment de Noël avec du maïs jeune.

On constate que le haricot, dont il existe dans la région un grand nombre de variétés d'origines très diverses ${ }^{15}$, peut entrer dans de nombreuses préparations culinaires à base de maïs. Ainsi l'apport en glucides du maïs est-il complété par l'apport protidique des haricots, ce qui donne lieu à une alimentation assez équilibrée, d'autant que la matière grasse est procurée par la viande ou le lait, ou par la margarine dans laquelle on a fait revenir les oignons avant de les incorporer au plat. Mais les Rwa cultivent aussi les haricots pour la vente, alors qu'en général la totalité de la production de maïs est réservée à la consommation familiale.

\section{La symbolique et les interdits alimentaires}

Les Rwa font une distinction entre les nourritures tendres (shoonga shiniiRi), c'est-à-dire faciles à digérer, recommandées ou réservées aux

15. G. PHILIPPSON (1984 : 153-154) individus faibles ou fragiles, et les nourritures dures (shoonga shuити), moins digestes à leurs yeux. Parmi les nourritures tendres figurent bouillie d'éleusine au lait ( $u R u)$, le $n s w a$ la pomme de terre, et tous les plats à base de viande bouillie, tels que le matendera (ragoût de viande et de banane verte bouillie), le kideri (plat réservé aux hommes d'âge mûr, à base de viande grasse, de banane et d'eau), ou l'usandiko (viande cuite dans une grande quantité de graisse). Le déclin de la richesse en bétail explique l'abandon actuel de cette dernière recette. Les nourritures dures, pour leur part, sont la viande rôtie et les plats de maïs à consistance plus ferme: ndolo, ngaRaRimu, ugali.

Les interdits alimentaires, très forts autrefois, ne sont plus guère respectés de nos jours, au grand dam des anciens qui regrettent amèrement la perte des traditions et le manque de respect des jeunes envers leurs aînés. Le principal interdit, encore actuellement, porte sur la consommation de légumes verts par les hommes après la circoncision, mais aucun informateur ne connaissait la raison d'être de cette prohibition. D'autres règles subsistent en principe, comme l'interdiction faite à la fiancée de donner à son futur mari une autre nourriture que de couleur blanche : elle devra se limiter essentiellement au ndolo avec du lait.

Les règles de commensalité autrefois plus strictes perdent, elles aussi, de leur vigueur. $\mathrm{Si}$ des facteurs individuels interviennent, certaines personnes étant plus soucieuses que d'autres de respecter les règles, les facteurs géographiques (la proximité de la ville d'Arusha) et le niveau d'éducation jouent un rôle indéniable. Il est encore de nombreuse familles où hommes et femmes mangent séparément, et où seuls les petits enfants peuvent partager le plat de leur parent de sexe opposé. Quant aux jeunes gens, après leur circoncision, ils éviteront de manger en présence d'une jeune fille qui n'est pas leur parente.

\section{Les modalités du changemen}

Au fil du siècle écoulé, l'alimentation des Rwa a beaucoup changé Elle s'est diversifiée, notamment en raison de l'influence de la ville. Avec la croissance démographique, les terres ne sont plus suffisantes pour assurer un revenu à tous et beaucoup de Rwa cherchent un complément de ressources ou une alternative dans un emploi salarié. En ville, ils se familiarisent avec d'autres pratiques alimentaires : galettes de maïs ou de blé (chapati), riz pilaf et ugali. La viande, par contre, se fait plus rare, en raison de la diparition des pâturages au profit des cultures.

Mais certains aliments résistent mieux que d'autres au changement. ''est le cas de l'éleusine, céréale qui conserve une fonction rituelle sociale très marquée, que ce soit sous forme de bouillie ou de bière banane. La consommation de bouillie d'éleusine, par l'accouchée et ses 
visiteurs, reste une tradition très observée chez les Rwa. Quant à la bière de banane, autrefois offerte et bue en toutes sortes de circonstances rituelles ou non, ith l'inflence n'a cessé de crôte sur le Mont Méru depuis l'art des premiers missionnaires allemands au début du $\mathrm{Xx}^{\mathrm{e}}$ siècle. Beaucoup d'hommes rwa continuent d'en consommer journellement de grandes quantités, mais d'autres, de plus en plus nombreux, s'en abstiennent, et cette bière tend à ne plus être offerte aujourd'hui lors des mariages religieux. Elle est alors remplacée par des sodas en bouteille, Pepsi ou Mirinda.

Le maïs, pour sa part, demeure une céréale uniquement alimentaire, mais elle se consomme sous des formes nouvelles. Le nswa, nourriture traditionnelle des Rwa, est de plus en plus considéré comme une nourriture du passé. On conserve pour lui une certaine tendresse, mais en même temps on en a un peu honte, comme de quelque chose qui n'est pas suffisamment moderne. De fait, il est largement abandonné de nos jours. Disparu des repas publics, il perd aussi du terrain dans les foyers au profit de l'ugali, pâte compacte de maïs mangée par tous les Tanzaniens. Les Rwa affichent ainsi leur caractère cosmopolite, leur appartenance au monde actuel. C'est d'ailleurs dans le même esprit que beaucoup de jeunes Rwa abandonnent leur propre langue, le ki-rwa, au profit du swahili national.

Mais d'autres raisons plus concrètes contribuent aussi à l'abandon du swa au profit de l'ugali. Il est maintenant facile et peu onéreux d'obteni de la farine de maïs, car les moulins où l'on peut apporter son grain à moudre pour un coût modeste sont plus répandus qu'autrefois. Or le recours à ce service facilite grandement la préparation du repas, car l'ugali est bien plus simple et plus rapide à préparer que le nswa. après trempage après trenpage et la très longue cuisson nécessaire pour les plats à base de maïs en grains. À ces économies de temps et d'efforts pour la ménagère s'en ajoute une troisième, celle réalisée sur le combustible. Car dans la plupart des foyers, c'est encore le bois qui est utilisé pour la cuisson des aliments, alors que son approvisionnement pose des problèmes croissants. Il faut aller de plus en plus loin le chercher, à moins qu'on ne l'achète sous forme de charbon. La dépense pour la mouture du grain es donc compensée aussi par l'économie de combustible.

Chez les Rwa du Mont Méru, les facteurs économiques se conjuguent ainsi aux facteurs psychologiques pour faire basculer la vie sociale vers un abandon de plus en plus rapide des traditions, notamment alimentaires. Dans ce domaine, l'éleusine, seule composante de base de l'alimentation des Rwa qui soit proprement africaine, apparait aussi comme le noyau des résistances les plus fortes, que ce soit sous forme de bouillie pour l'accouchée ou de bière de banane consommée surtout par les hommes.

\section{Bibliographie}

BAHUChet S. et PHILIPPSON G., 1998, «Les plantes d'origine américaine en Afrique bantoue: une approche linguistique», in CHASTANET M. (éd.), Plantes et paysages d'Afrique. Une histoire à explorer, Paris, Karthala-CRA : 87-116.

BAROIN C., à paraître, «Une chefferie «traditionnelle » réinventée : les Rwa du Mont Meru (Tanzanie du Nord)», in Perrot C.-H. FAUVELLE-AYMAR F.-X. (eds), Le retour des rois. Les autorités traditionnelles et l'État en Afrique contemporaine, Paris, Karthala, sous presse.

ChRÉTIEN J.-P., 1998, «Anciens haricots et anciens tubercules dans la région des Grands Lacs », in Chastanet M. (éd.), Plantes et paysages d'Afrique. Une histoire à explorer, Paris, Karthala-CRA : 213-229.

KAAYA B., 1978, «The planting of Christianity in Meru. Its conflicts and similarities with the traditional culture of the Wameru », Kampala, Makerere University, Diploma in theology.

MOORE S.F., 1986, Social facts and fabrications. «Customar » law on Kilimanjaro, 1880-1980, Cambridge, Cambridge University Press.

Philippson G., 1984, «Gens des bananeraies » (Tanzanie). Contribution linguistique à l'histoire culturelle des Chaga du Kilimanjaro, Paris, Éditions Recherche sur les Civilisations.

RAum O.F., 1940, Chaga childhood. A description of indigenous education in an East African tribe, Oxford, Oxford University Press for the International African Institute, reéd. 1967.

SPEAR T. (éd.), 1995, Evangelisch-Lutherisches Missionsblatt. Extracts on Arusha and Meru, 1897-1914, [trad. C. M. et T. M. Murphy], Madison, University of Wisconsin-Madison, African Studies Program.

Von HöHNEL L., 1968, Discovery of Lakes Rudolf and Stefanie. A Narrative of Count Samuel Teleki's exploring and hunting expedition in Eastern Equatorial Africa in 1887 and 1888, [trad. N.Bell], Londres, Frank Cass \& Co., 2 vol. 